\title{
INVESTIGATING THE PERFORMANCE OF VARIOUS VOCODERS FOR A FAIR SCHEDULING ALGORITHM IN WIMAX
}

\author{
B. Kaarthick Member IEEE ${ }^{1}$, V. J.Yeshwenth ${ }^{2}$, P.M.Sudarsan ${ }^{3}$, N.Nagarajan ${ }^{4}$, \\ Rajeev Senior Member IEEE ${ }^{5}$ \\ ${ }^{1}$ Network System Design Center, Sri Krishna College of Engineering \& Technology, \\ Coimbatore, India. \\ kaarthick_cbe@yahoo.com \\ ${ }^{2,3}$ University Of Texas at Dallas, USA. \\ yeshwenthegmail.com \\ ${ }^{4}$ Coimbatore Institute of Eng. and Information Technology, Coimbatore. \\ ${ }^{5}$ Wipro Technologies,India \\ rajeev@ieee.org
}

\begin{abstract}
IEEE 802.16e, a broadband wireless access technology that provides high speed internet access and multimedia services has seen very significant growth in recent times. With the due rapid deployment of WiMAX, there would be a growing demand for efficient support of voice applications. The performance of Voice over IP services depends on a proper scheduling algorithm combined with a proper Voice encoder scheme. The contribution of this work is two fold. The first is that we present an efficient scheduling algorithm that can be used for VoIP services in IEEE 802.16e. We then implement the algorithm in various CODECS and compare the performance of the codec's i.e.G.728, G.711, G.723, G.729, G.726, GSM-EFR, GSM-HR under similar load conditions for Interactive voice over WiMAX by enabling the Speech Activity Detection (SAD). We analyze the system throughput and delay to evaluate the performance of our algorithm and the primary metrics that were used in the comparison for choosing the right vocoders for the algorithm were Jitter, MOS, and packet end to end delay.
\end{abstract}

\section{KEYWORDS}

QoS; vocoders; Jitter; VoIP; MOS

\section{INTRODUCTION}

The WiMAX (Worldwide Interoperability for Microwave Access) is based on IEEE 802.16 wireless Metropolitan Area Network standard and has been deployed extensively in recent years to solve the problems associated with point-to-multipoint broadband outdoor wireless networks [1]. The 802.16 standard provides numerous inventive features enabling high traffic rates, bounded delays, flexible and scalable system architecture, which makes it commercially attractive for various broadband wireless services. Voice over IP over WiMAX, is regarded to become a killer application in IEEE 802.16e which aims at providing multimedia Application services [2], [3].

Traditional scheduling algorithms [4], [5], [6] in WiMAX tend to penalize the Subscriber Stations that are situated far off from the Base Station with lower coding rates because the channel quality degrades with distance. To pose the problem in a right perspective, the mobile stations near the BS always enjoy higher data rate without ignoring the fairness criteria. As we move away from BS, a change in modulation and coding scheme will be towards higher 
modulation format and lower coding rate. This is because the channel quality tends to degrade as the SS move away from BS. As a result, the mobile stations away from the BS are always penalized.

In this paper, we present a novel scheduling algorithm which would re-allocate resources in a dynamic and fair manner. The reallocation is carried out by few modifications in MDRR algorithm and considering a single cell as different regions. Calculating the channel quality and $\mathrm{n}$-factor does the reallocation of resources. In mobile WiMAX, a Channel Quality Indicator Channel (CQICH) is dedicated to return channel quality measurements, measured by the SS to the BS. The parameters sent over this channel are the CINR and RSSI value.

To transmit Voice over IP through any network, the voice signal is encoded at the transmitter side and then transmitted to the receiver through the communication channel. At the receiving end, the encoded signal is then decoded and reconstructed. A number of vocoders are available for voice transmission over wireless channels and every vocoder is unique.

The G.711 codec was the first ITU-T recommended Speech coder. It supports a Bit rate up to $64 \mathrm{kbps}$. The G.728 is an ITU-T standard codec, which operates at $16 \mathrm{kbps}$. It is widely used for applications that require very low algorithmic delay. G.723.1 is a dual-rate speech codec developed for videophones that deliver video and speech over regular phone lines (PSTN). The codec operates at 5.3/6.3kbps. The G.729 is based on Code Excited Linear Prediction (CELP) coding model.G.729 is the lowest bit rate ITU-T standard with a bit rate of 8 kbps. The G.726 supports varied transmission rates of 16,24, 32 and 40. GSM-EFR is another audio compression scheme operating at $12.2 \mathrm{kbps}$, which is optimized for providing improved speech quality and better robustness to network impairments. The GSM-HR codec supports a transmission rate of $6.5 \mathrm{kbps}$ and was developed with a special purpose of increasing network capacity [7], [8].

A VoIP application usually works as follows. The voice signal is sampled, digitized, and encoded using a given algorithm/coder. Using RTP/UDP/IP the encoded data is packetized and transmitted. At the receiver's side, data is de-packetized and forwarded to a play out buffer, which smoothes out the delay incurred in the network [9]. Finally, the data is decoded and the voice signal is reconstructed. The quality of the reconstructed voice signal is subjective and therefore is measured by the mean opinion score (MOS). MOS is a subjective quality score that ranges from 1 to 5.As the VoIP packets permeates from the backbone network to the WiMAX network the quality of VoIP packets tend to decrease due to the difference in the characteristics of wired and wireless networks [10]. The key performance metrics that impact the voice quality in wireless channels are Jitter and end to end delay .

The objective of this research work is to evaluate the performance of various vocoders by implementing the proposed scheduling algorithm in the various vocoders. We compare the performance of different Vocoders based on MOS, end to end delay and jitter. Based on the simulation results, we suggest a suitable vocoder that is best suited for the algorithm.

We first introduce our proposed scheduling algorithm for mobile WiMAX networks in section 2. In section 3, we give the detailed description of the simulation scenario. In section 4 we evaluate the performance of the algorithm. Finally we summarize the paper in section 5 .

\section{PROPOSED ALGORITHM}

For the sake of conceptual clarity, we first review the MDRR algorithm and describe later, our proposed algorithm. The queuing mechanism adopted in CISCO 12000 series of routers is referred to as Modified Deficit Round Robin (MDRR). Absence of the low-latency queue in DRR makes it different from the MDRR. Each queue within MDRR is defined by two variables:

Quantum value: This is the average number of bytes served in each round

Deficit counter: This counter is used to track how many bytes a queue has transmitted in each round. It is initialized to the quantum value.

In the MDRR algorithm, there are $\mathrm{n}$ numbers of queues and each queue is accompanied by a deficit counter which is initialized with a quantum value. In round robin fashion, each non- 
empty queue is served when the deficit counter is greater than zero. A queue can be served when the deficit counter is positive; otherwise it is not possible to serve a queue when counter value becomes zero or negative. In each round, the deficit counter is incremented by a quantum value. In order to serve atleast one packet in each round, the quantum size should not be smaller than maximum transmission unit (MTU). Assigning a weight to each queue is helpful in MDRR when the interface is congested.

In normal MDRR, fixed weight is allocated to each connection. Service class scheduling in IEEE 802.16e becomes very difficult because of the fixed weight assignment used, which results in QoS degradation. In our algorithm, we adopt a dynamic weight assigning strategy which helps to control the coding rate and also helps to manage the overall system capacity. The queue is prioritized depending upon the weight assigned to them. The different service flows are assigned different weights using our strategy.

Our algorithm starts the initialization process by verifying the default queue. Scheduling process is invoked for a non-empty queue. Type of Service (ToS) based scheduling is employed in the algorithm. Once the scheduling algorithm is invoked, it starts functioning like a normal MDRR. In the first round, the pointer moves for queue 1 to $n$ servicing the queues having quantum values less than the deficit counter. At the end of the first round, CINR value is obtained from the channel to calculate the new quantum value. The CINR is substituted in (1), to obtain new quantum value. Further, the CINR value is compared with the pre-assigned threshold. When the CINR value is below the threshold, change of modulation has to take place. Modulation technique change may cause the data rate to increase or decrease whether the mobile station is either moving towards or away from the BS. The variation in CINR may be attributed temporarily due to several factors and hence monitoring the value of CINR over a period of time is needed before switching to the other modulation schemes. During this waiting period the packets may be lost, so reducing the weight will ensure the proper delivery of packets even if it as with less data rates. The waiting period happens for five rounds of servicing before change in modulation takes place. Frequent change in modulation schemes will also burden the service station. A unified modulation technique is the best outcome for the above specified problem.

The second phase of the algorithm, calculates the n-factor. Each cell is divided into three regions based on the distance between BS and geographical location. Each region is assigned a threshold CINR value. The $\mathrm{n}$ factor is the number of subscriber stations falling in each region. Usually, the subscriber stations in the outermost region or which is farthest away from the base station will have the lowest CINR. These SS requires a robust modulation scheme which in turn will decrease the data rate. Hence, it always becomes a practice to penalize the far off stations with low coding rates. In our algorithm, we receive the $\mathrm{n}$-factor information from the BS and reallocate resources for a fair scheduling.

The SS near the BS (region 1) enjoy high data rates because of the high CINR thereby it consumes more resources. The number of subscribers in each region is monitored regularly at the end of each round in the proposed algorithm. As the number of SS in region 3 increases over other regions drastically, a unified modulation scheme is employed for both region 1 and region 2. This ensures that the resources are uniformly distributed in region 1 and region 2, thereby reducing the discrepancy in bandwidth allocation. The subscribers in region 1 continue to work in higher data rates. A similar strategy is followed between region 1 and region 2 . The algorithm is explained using max-plus and min-plus algebra of network calculus. Network calculus is preferred in our algorithm because it is best suited for pre-assigned thresholds.

The capacity of the queue will vary with time in the network, $\mathrm{h}$ is assumed for the packets in queue at time $t$, $f$ denotes the packets arrival at time $t$ and $g$ is defined in WiMAX which is a constant,

$$
\begin{aligned}
& (f+h)(t)=f(t)+h(t) \\
& (f \wedge h)(t)=f(t) \wedge h(t)
\end{aligned}
$$

And $\Pi(\mathrm{x})$ is the CINR assigned to each round which varies as a function of distance $\mathrm{x}$ 
Quantum value: $q_{i}=A \wedge 512 * w(x, g)(1) \quad$ Where $w(x, g)=g+\Pi(\mathrm{x})$ obtained after extensive simulation/

/ $d_{i}-$ deficit counter $/$

$$
q_{i}=q_{i} \wedge d_{i}
$$

If $d_{i}>0$

Do

Serve packet

/C - packet size (can be variable) /

$$
d_{i}=d_{i}-c
$$

If $\pi(x)<\lambda$

/A - threshold limit for CINR in each region/

$$
(f \varnothing h)(t)=\inf \{f(t+w)-g(w)\}
$$

/ determines packet delivery /

$$
\mathrm{u} \geq 0
$$

/ terminates this loop upon satisfying this condition /

$$
\begin{gathered}
(f \otimes g)(t) \\
=\sup [f(t-w)+g(u)] \\
0 \leq \mathrm{s} \leq \mathrm{t}
\end{gathered}
$$

The $\mathrm{n}$-factor which is described in the algorithm is used here:

$$
w(x, g)=\sup \Pi\left[x_{n}\right]
$$

$\mathrm{n}$

/ Weight is calculated with the obtained CINR /

$$
\begin{aligned}
\left(h \cdot h^{\prime \prime}\right)(t, w) & =\inf \left[h(t, w)+h^{\prime \prime}(t, u)\right] \\
\mathrm{u} & \square \mathrm{s}
\end{aligned}
$$

/ assuring proper delivery of packets /

/ duration which the CINR is monitored /

$$
\begin{aligned}
\Pi(\mathrm{x})(\mathrm{t}) & =\operatorname{jnf}\left\{\min \left\{\mathrm{h}_{\mathrm{i}}\left(t_{\mathrm{s}} \mathrm{s}\right)+\mathrm{x}_{\mathrm{i}}(\mathrm{s})\right\}\right] \\
\mathrm{s} & \square \mathrm{t} \quad 1 \leq \mathrm{i} \leq \mathrm{J}
\end{aligned}
$$

/ choosing the modulation scheme /

$$
\overline{\left(\Pi \wedge h_{n}\right)}=\overline{\left(h_{n} \cdot \Pi\right)} \cdot h_{n}
$$

\section{EXPERIMENTAL SETUP}

Fig. 1 illustrates the network model. The cell is mapped into three regions with the base station at the centre and 16 subscriber stations. The uplink network configuration parameters are shown in Fig. 2. The cell is divided into regions 1,2 and 3 as explained earlier. The threshold levels for each region are assigned. Trajectory is set for the SS nodes to make them mobile as illustrated in Fig. 1. The performance of the algorithm is tested on various SS but the analysis in this paper deals with results obtained for node (mobile_1_2). The Call volume is set as 1000 Erlangs and the Average call duration is 300 seconds per call. We have selected Interactive voice as the type of service. In each simulation a specific voice coder is tested. The simulation is carried out by enabling the SAD and the performance of the codec is evaluated. The same procedure is repeated for other codecs. 


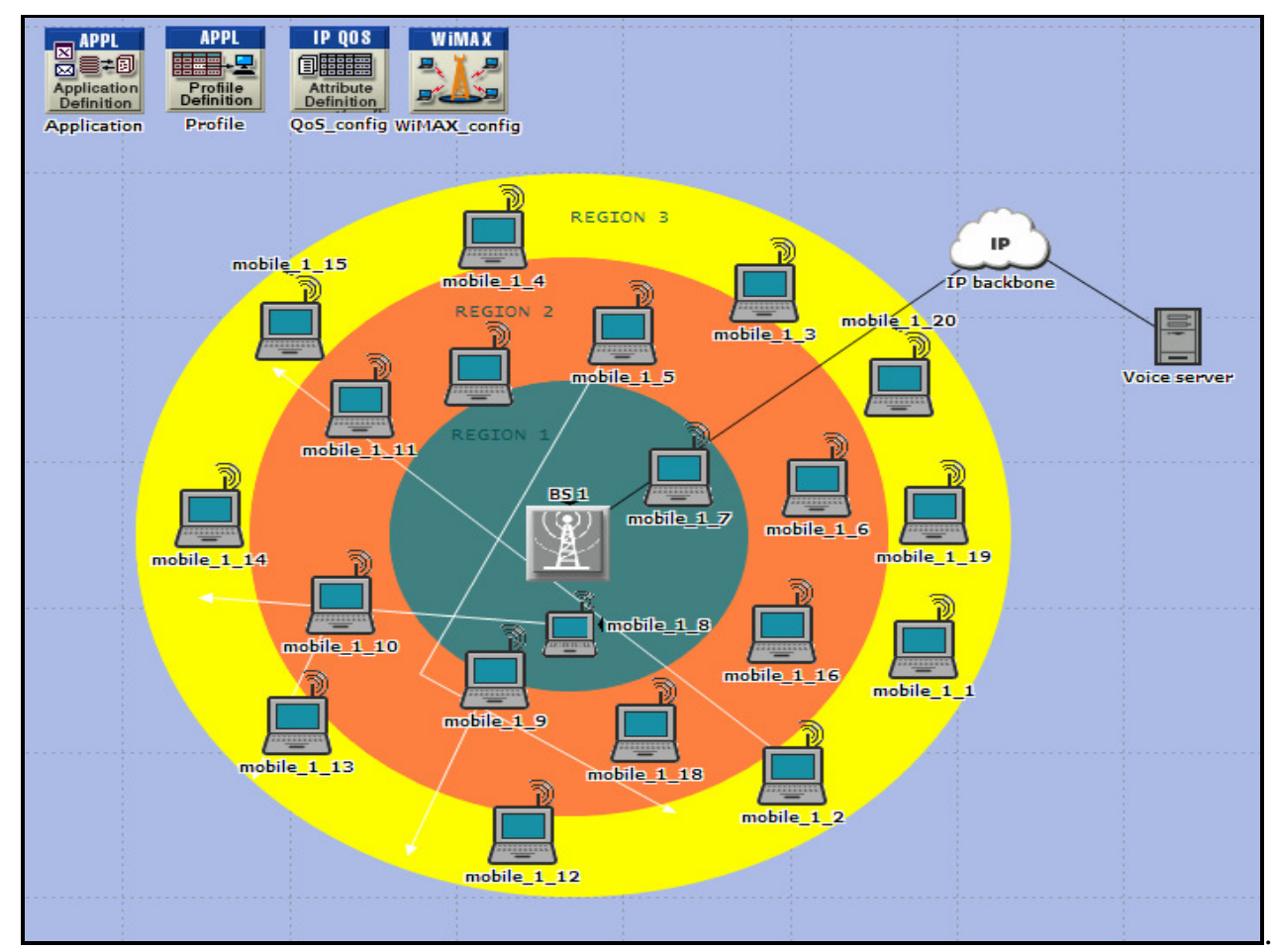

Figure 1. Network Model

\begin{tabular}{|c|c|c|c|c|c|c|}
\hline \multicolumn{4}{|c|}{ * (BS 1) Attributes } & & $\square$ & $x$ \\
\hline \multicolumn{7}{|c|}{ Type: router } \\
\hline \multicolumn{2}{|r|}{ Attribute } & \multicolumn{2}{|l|}{ Value } & & & $\Delta$ \\
\hline \multirow{2}{*}{\multicolumn{2}{|c|}{$\begin{array}{l}\text { \& A,U-HUL Houting Parameters } \\
\text { † ARP }\end{array}$}} & & & & & \\
\hline \multirow{2}{*}{\multicolumn{2}{|c|}{$\begin{array}{l}\text { } \text { ARP } \\
\text { Ð ATM-IP Interface }\end{array}$}} & & & & & \\
\hline & & & & & & \\
\hline \multicolumn{2}{|r|}{$\oplus \mathrm{ATM}$} & & & & \\
\hline \multicolumn{2}{|c|}{ (3) Address } & & & & & \\
\hline \multicolumn{2}{|c|}{$\because$ WiMAX Parameters } & \multicolumn{2}{|c|}{ Auto Assigned } & & & \\
\hline & Antenna Gain [dBi] & \multicolumn{2}{|l|}{$15 \mathrm{dBi}$} & & & \\
\hline & Æ BS Parameters & \multicolumn{2}{|l|}{$[\ldots]$} & & & \\
\hline & $\boxminus$ Classifier Definitions & \multicolumn{2}{|l|}{$[\ldots]$} & & & \\
\hline & Number of Rows & \multirow{2}{*}{\multicolumn{2}{|c|}{1}} & & & \\
\hline \multicolumn{2}{|r|}{$\Theta$ Row 0} & & & & & \\
\hline (?) & Type of SAP & \multicolumn{2}{|l|}{ IP } & & & \\
\hline (?) & $\Theta$ Traffic Characteristics & \multicolumn{2}{|l|}{$\begin{array}{l}\text { [...] } \\
\text { IP ToS }\end{array}$} & & & \\
\hline (?) & Match Property & \multicolumn{2}{|l|}{ IP ToS } & & & \\
\hline (?) & Match Condition & \multicolumn{2}{|l|}{ Equals } & & & \\
\hline (?) & Match Value & \multicolumn{2}{|c|}{ Interactive Voice [6] } & & & \\
\hline (?) & Service Class Name & \multicolumn{2}{|c|}{ Gold } & & & \\
\hline (?) & MAC Address & \multicolumn{2}{|c|}{ Auto Assigned } & & & \\
\hline (?) & - Maximum Transmission Power [W] & \multicolumn{2}{|l|}{0.5} & & & \\
\hline (?) & PHY Profile & \multirow{2}{*}{\multicolumn{2}{|c|}{ WirelessDFDMA. $20 \mathrm{MHz}$}} & & & \\
\hline \multirow[t]{2}{*}{ (3) } & PHY Profile Type & & OFDM & & & - \\
\hline \multirow{2}{*}{\multicolumn{2}{|c|}{ (?) }} & \multirow{3}{*}{ Filter } & & ГА & dyar & aced \\
\hline & & & ГАpp & electe & d obi & ects \\
\hline \multicolumn{2}{|c|}{ Г Exact match } & & $\underline{\underline{K}}$ & & ancel & \\
\hline
\end{tabular}

Figure 2. BS Parameters 


\section{RESULTS}

We first evaluate the performance of the scheduling algorithm on the overall throughput and delay of the network and then proceed to the evaluation of the various vocoders implementing the algorithm.

\subsection{Evaluation of the Algorithm}

From the study of the graph of overall throughput of the system it may be noted that the overall throughput of the WiMAX network has improved by approximately 35\% (Fig.3). Fig.4 illustrates the average delay experienced by the network. Average delay is the end-to-end delay of all the packets received by the WiMAX MACs of all WiMAX nodes in the network and forwarded to the higher layer. It may be noted that our algorithm provides better or equivalent performance compared to the conventional.

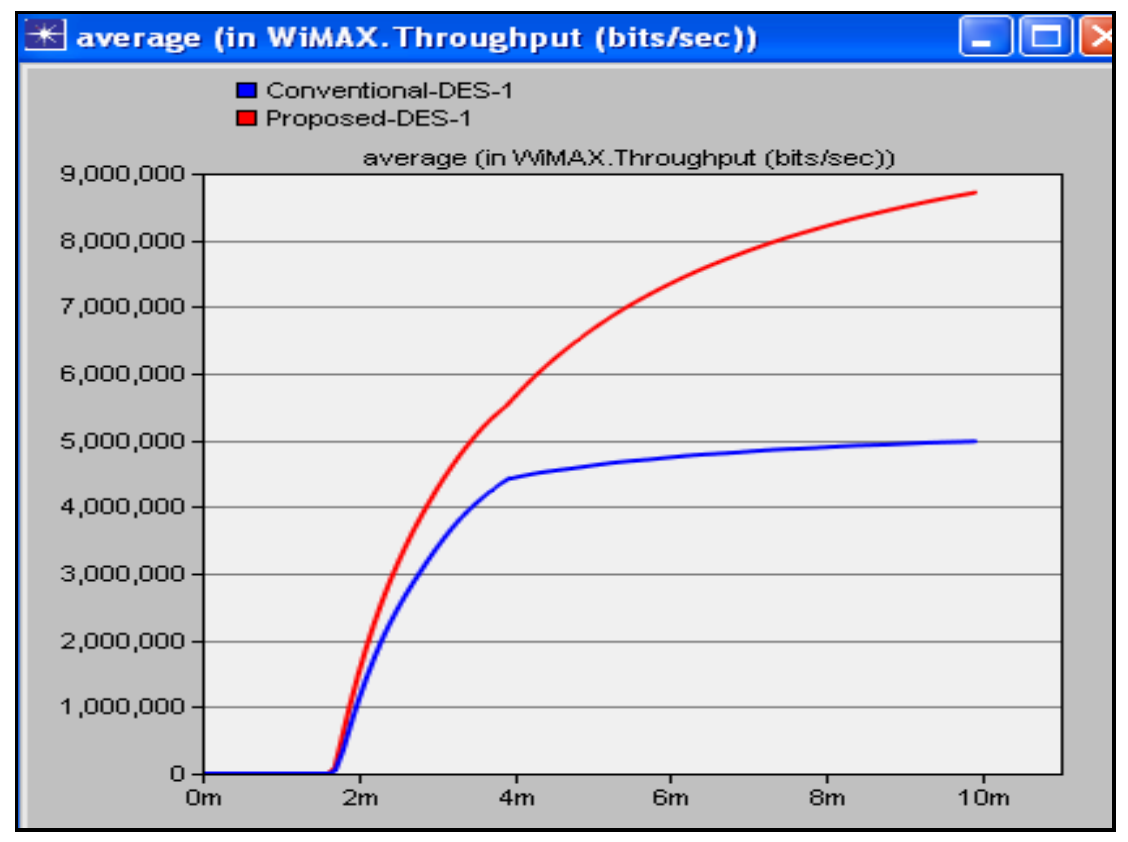

Figure 3. WiMAX Throughput (bits/sec) 


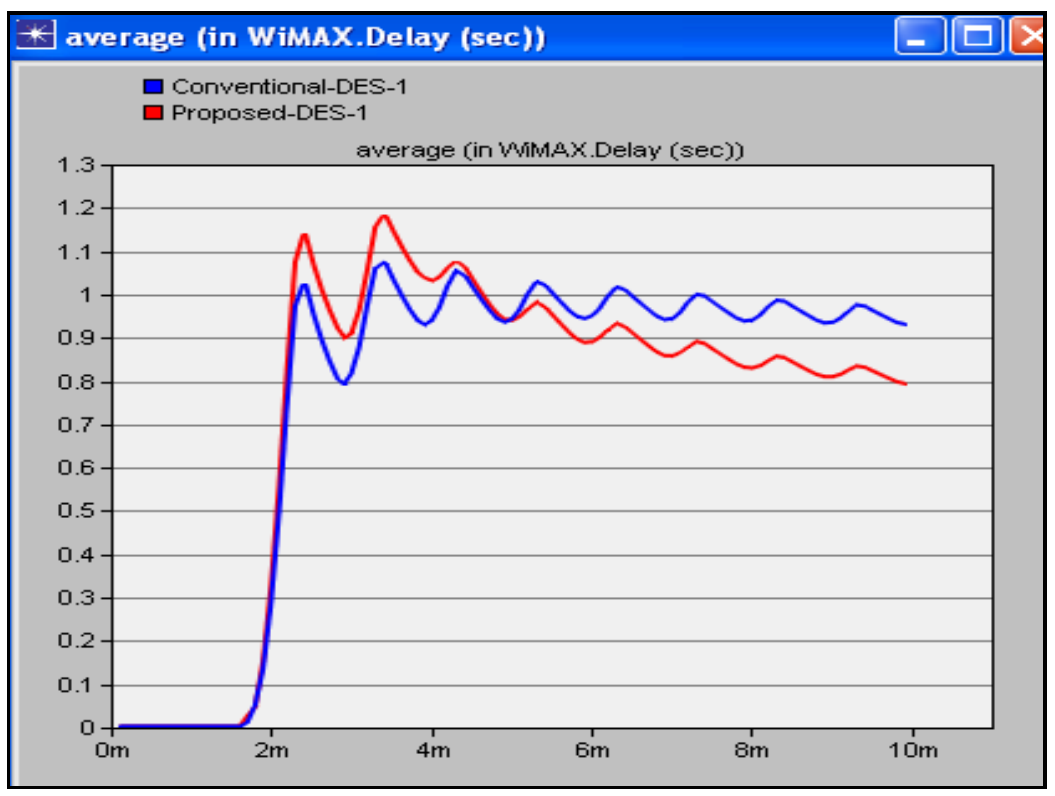

Figure 4. WiMAX delay (sec)

\subsection{Evaluation of Vocoders}

The algorithm was tested for seven different Vocoders. During the simulation we have enabled the Speech Activity Detection (SAD) for the Vocoders and analyzed the performance of the Vocoders in terms of Packet end to end delay, jitter and MOS.

Table 1 illustrates the comparison of the various vocoders implementing the conventional and proposed scheduling algorithm. G.728 provides the least packet end to end delay and a further improvement of $6.29 \%$ in end to end day is achieved by implementing our algorithm. GSMEFR provides the maximum packet end to end delay of $25 \mathrm{~ms}$ but by using our algorithm the delay has been reduced by $12.8 \%$.Analysis has also been done for jitter and it shows that the GSM-EFR and G.723 are better. Further, analyzing the MOS values we find that the GSM-EFR provides better MOS than the other Vocoders. Fig 5 and Fig 6 demonstrates the Packet end to end delay and jitter of the various Vocoders. The GSM-EFR with the proposed scheduling algorithm provides an end to end delay of $21.80 \mathrm{~ms}$ which is comparable to the G.728 standard but the GSM-EFR outperforms all other Vocoders in terms reduced Jitter and improved MOS. Hence we suggest that the GSM-EFR is best suited for Voice over IP communication in WiMAX. 


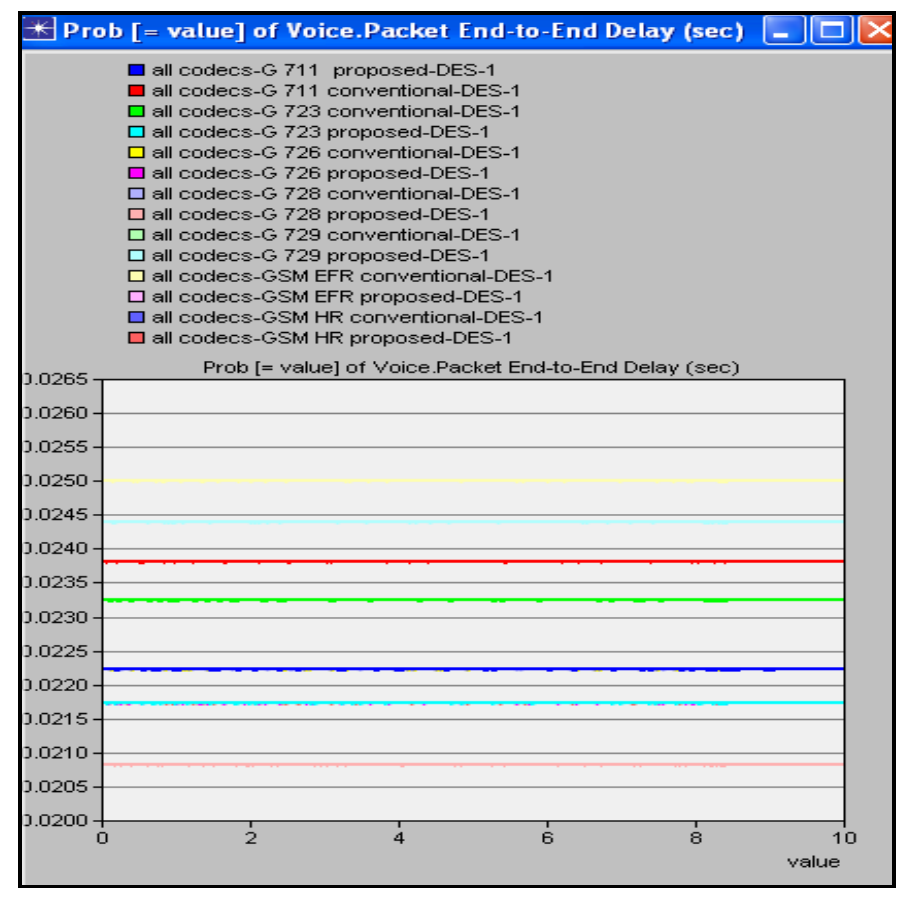

Figure 5. Packet end to end Delay

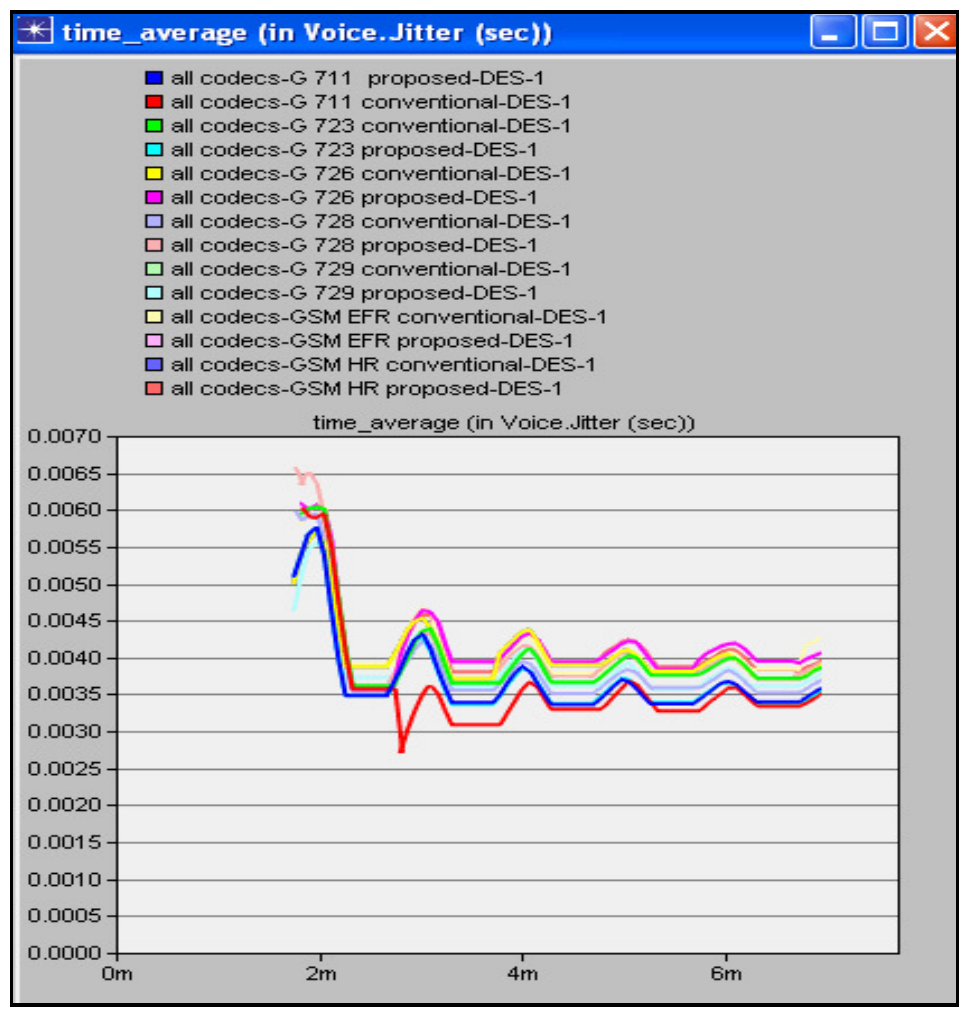

Figure 5. Voice Jitter 
Table 1. Comparison of Average end to end delay, Jitter, MOS for WiMAX Standard

\begin{tabular}{|c|c|c|c|c|c|c|c|}
\hline S.No & $\begin{array}{l}\text { Name of } \\
\text { Vocoder }\end{array}$ & $\begin{array}{l}\text { Speech } \\
\text { Activity } \\
\text { Detection }\end{array}$ & $\begin{array}{l}\text { Average } \\
\text { packet } \\
\text { end to } \\
\text { end } \\
\text { delay }(\mathrm{ms})\end{array}$ & Jitter & MOS & $\begin{array}{l}\text { Reduction } \\
\text { in packet } \\
\text { end to } \\
\text { end } \\
\text { delay }(\mathrm{ms})\end{array}$ & $\begin{array}{l}\% \\
\text { Improvement }\end{array}$ \\
\hline \multirow{2}{*}{1} & \multirow{2}{*}{ G.728 } & Proposed & 20.85 & 0.0035 & 2.6 & \multirow{2}{*}{1.4} & \multirow{2}{*}{6.29} \\
\hline & & Conventional & 22.25 & 0.0040 & 2.4 & & \\
\hline \multirow{2}{*}{2} & \multirow{2}{*}{ G.711 } & Proposed & 22.2 & 0.0033 & 2.7 & \multirow{2}{*}{1.6} & \multirow{2}{*}{6.72} \\
\hline & & Conventional & 23.8 & 0.0036 & 2.5 & & \\
\hline \multirow{2}{*}{3} & \multirow{2}{*}{ G.723 } & Proposed & 21.75 & 0.0030 & 2.7 & \multirow{2}{*}{1.5} & \multirow{2}{*}{6.45} \\
\hline & & Conventional & 23.25 & 0.0035 & 2.3 & & \\
\hline \multirow{2}{*}{4} & \multirow{2}{*}{ G.729 } & Proposed & 23.3 & 0.0033 & 2.9 & \multirow{2}{*}{1.1} & \multirow{2}{*}{4.50} \\
\hline & & Conventional & 24.4 & 0.0035 & 2.7 & & \\
\hline \multirow{2}{*}{5} & \multirow{2}{*}{ G.726 } & Proposed & 21.75 & 0.0035 & 2.5 & \multirow{2}{*}{0.5} & \multirow{2}{*}{2.24} \\
\hline & & Conventional & 22.25 & 0.0038 & 2.3 & & \\
\hline \multirow{2}{*}{6} & GSM- & Proposed & 21.8 & 0.0030 & 3.3 & \multirow{2}{*}{3.2} & \multirow{2}{*}{12.8} \\
\hline & EFR & Conventional & 25.0 & 0.0035 & 3.1 & & \\
\hline \multirow{2}{*}{7} & GSM- & Proposed & 21.75 & 0.0037 & 2.9 & \multirow{2}{*}{0.45} & \multirow{2}{*}{2.02} \\
\hline & HR & Conventional & 22.20 & 0.0034 & 2.4 & & \\
\hline
\end{tabular}

\section{CONCLUSIONS}

In this paper, we have presented a new scheduling algorithm, which provides fair resource scheduling for all the users in the cell. The algorithm provides resource reallocation based on CINR value from CQICH and $n$-factor. We experimentally compared the performance of the algorithm with the conventional MDRR algorithm and have shown that our algorithm consistently performs better than the conventional algorithm.

We also compared and analyzed the performance of seven Vocoders (G.728, G.711, G.723, G.729, G.726, GSM-EFR, GSM-HR) under similar load conditions in a WiMAX environment using our algorithm. We have identified that the GSM-EFR outperforms the other voice coders and is best suited for Voice over IP over a WiMAX network. We have identified that the GSMEFR outperforms the other voice coders and is best suited for Voice over IP over a WiMAX.

\section{REFERENCES}

[1] IEEE Std 802.16-2004, IEEE Standard for Local and Metropolitan Area Networks, Part 16: Air interface for Fixed Broadband Access Systems, October, 2004.

[2] IEEE Standard for Local and Metropolitan Area Networks - Part 16: Air Interface for Fixed and Mobile Broadband Wireless Access Systems Amendment 2," Feb. 28.

[3] Jeffrey G.Andrews, Arunabha Ghosh, Rias Muhammed "Fundamentals of WiMAX Understanding Broadband Wireless Networks" Prentice Hall Inc, 2007. 
[4] Belghith, A. Nuaymi, L. ENST Bretagne and Rennes, "Comparison of WiMAX scheduling algorithms and proposals for the rtPS QoS class," This paper appears in: Wireless Conference, 2008. EW 2008. 14th European, Publication Date: 22-25 June 2008, pp 1-6

[5] LEE Howon, KWON Taesoo, CHO Dong-Ho, "An efficient uplink scheduling algorithm for VoIP services in IEEE 802.16 BWA systems," Vehicular Technology Conference, 2004. VTC2004-Fall. 2004 IEEE $60^{\mathrm{th}}$, vol. 5, pp. 3070-3074.

[6] Jin-Cherng Lin, Chun-Lun Chou and Cheng-Hsiung Liu, "Performance Evaluation for Scheduling Algorithms in WiMAX Network," Advanced Information Networking and Applications Workshops, 2008. AINAW 2008. 22nd International Conference vol., Issue , 25-28 March 2008, pp. $68-74 \mathrm{M}$.

[7] http://en.wikipedia.org/wiki/list_of_codecs.

[8] Micheal Y.Appial, Raimonda Marrickalite, Milda Gusaite, Sasikanth Managala,'Robust Voice Activity Detection and Noise Reduction Mechanism using higher order stastistics",June-05.

[9] S. Sengupta, M. Chatterjee, S. Ganguly, "Improving Quality of VoIP Streams over WiMAX“, IEEE Transactions on Computers, Vol. 57, No.2, February.

[10] Hung-Hui Juan; Hsiang-Chun Huang, "Cross-layer System Designs for Scalable Video Streaming over Mobile WiMAX", IEEE Wireless Communications and Networking Conference , Page(s) 1860-1864, 11-15 March. 